\title{
The Role of 3D Imaging in the Practice of Medicine and Medical Education
}

\section{Chaya Prasad, MD, MBA ${ }^{1}$, ; Sharon Lee OMS-III²; Jenny Vang OMS-III²}

${ }^{1}$ Western University of Health Sciences, Department of Clinical Sciences, Pomona, CA

${ }^{2}$ Western University of Health Sciences, Pomona, CA

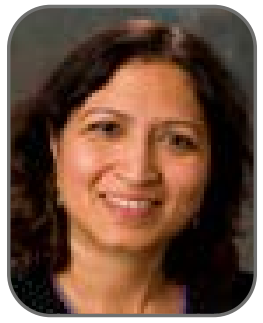

Chaya Prasad, MD, MBA

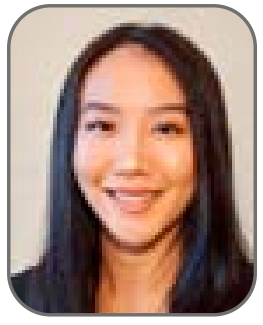

Sharon Lee, OMS-III

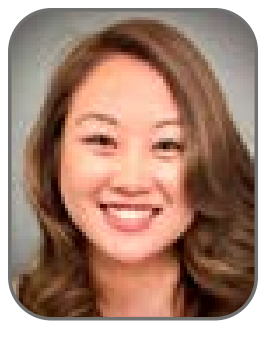

Jenny Vang, OMS-III

\section{CORRESPONDENCE:}

Chaya Prasad, MD, MBA

cprasad@westernu.edu

DOI: $10.38206 / 130102$

\section{KEYWORDS:}

3D Imaging, Laser Scanning, Practice of Medicine, Technology

\section{ABSTRACT}

Technology has revolutionized the practice of medicine. X-rays were the gold standard of imaging for many decades, but in recent years 3D imaging technology has been developed to represent the exact anatomic, pathologic disease processes. In medical practice, 3D images can be obtained by a variety of methodologies, including 3D laser scanning, computerized tomography (CT), and magnetic resonance imaging (MRI). Since 3D imaging technologies have been incorporated into the health field, it has made tremendous advancements in all aspects of medicine, including orthopedic surgery, neurovascular surgery, forensic pathology, pathology, and dentistry. 3D imaging can be used not only for diagnostic services, but also as treatment modalities. In addition, $3 \mathrm{D}$ imaging has also made an impact in medical and allied health education. From the 3D scanners used to produce optimal reconstruction of surgical pathology specimens to the fluoroscopic 3D imaging used to aid orthopedic surgeons in surgery strategies, the purpose of this review is to explore the current literature to illustrate the impact of $3 \mathrm{D}$ imaging on medical practice and to also discuss future applications.

\section{INTRODUCTION}

Technology has revolutionized the practice of medicine. We are dependent on technology in virtually every field of medicine. In 1895 when X-rays were introduced, it was the gold standard of imaging for many decades. ${ }^{1}$ Given that an $\mathrm{X}$-ray radiograph is a $2 \mathrm{D}$ image, there are certain limitations of capturing a $3 \mathrm{D}$ object in the two-dimensional field. Structures superimpose on each other, and it becomes difficult to appreciate the pathologic processes in situ. ${ }^{2}$ 3D imaging technology was developed to circumvent this issue and represent the model of the anatomy and pathological disease processes more accurately.
Current imaging methods include ultrasound, computed tomography (CT), and positron emission tomography -CT (PET-CT), all producing 2D images, but with the evolution of technology, the use of $3 D$ imaging has quickly established a prominent role in the practice of medicine. ${ }^{3}$ Given the potential of this growing field, literature on 3D imaging has increased exponentially over the years, illustrating the broad range of applications for the new technologies. From the 3D scanners used to produce optimal reconstruction of surgical pathology specimens to the fluoroscopic 3D imaging used to aid orthopedic surgeons in surgery strategies, the purpose of this review is to explore the 
current literature on how 3D imaging is currently impacting medical practice and secondarily and to discuss future applications of 3D imaging.

\section{HISTORICAL BACKGROUND}

3D imaging has become a critical emerging technology in the healthcare field. Many complex medical procedures, diagnoses, and treatments are possible today with the advancement of $3 \mathrm{D}$ imaging. In 1895, the first X-ray image of the human bones was reproduced by Wilhelm Roentgen and this revolutionized medicine. ${ }^{3}$ In 1972, X-rays CTs were introduced by Godfrey Hounsfeild in the medical field to noninvasively view the human body. ${ }^{4}$ CT scans generate images by using $X$-rays beams to scan the human anatomy while the recorded images are transmitted to a computerized system for radiologists to view. ${ }^{5}$ They have transformed our knowledge resulting in a deeper understanding of pathological processes, an important step in the diagnosis of diseases. Since its development, CT has greatly impacted the health field and today it has become the fundamental building block of 3D imaging.

In 1986, Charles Hull developed 3D laser scanning, another addition to 3D imaging, which has also impacted the healthcare field. ${ }^{6}$ 3D scanners use lasers to digitally recreate identical computerized images of the anatomical structure of interest. With the images from the 3D laser scan, clinicians are able to make an exact 3D anatomical replica through a 3D printer. Many of these anatomical replicas are useful to clinicians as it allows them to study the patient's anatomy in diseased states.

In 2006, 3D imaging led to the development of Multiple Detector Computer Tomography (MDCT), a 3D technology that uses $\mathrm{X}$-rays from CT scans to capture multiple crosssectional images and reconstructs them to form 3D images. ${ }^{7}$ MDCT has contributed to the advancement of our clinical knowledge in comparison to a single-detector $\mathrm{CT}^{7}$. For example, MDCT has allowed clinicians to view image slices of a patient's anatomy within minutes. It is capable of generating high resolution images with improved temporal and spatial depth. ${ }^{7}$ These images are stored in a Picture Archive and Communication System (PACS). PACS was developed in the $1990^{\prime}{ }^{8}$ as an image storing database which provides clinicians with a convenient interface to save, organize, and manipulate recorded images from many imaging technologies such as MDCT. The technologies described above have allowed for a rapid, non-invasive, and cost-effective 3D reconstruction of the anatomic structure. ${ }^{?}$

\section{FIGURE 1.}

A line of laser light projecting onto the surface, while sensors record displacement of laser light.

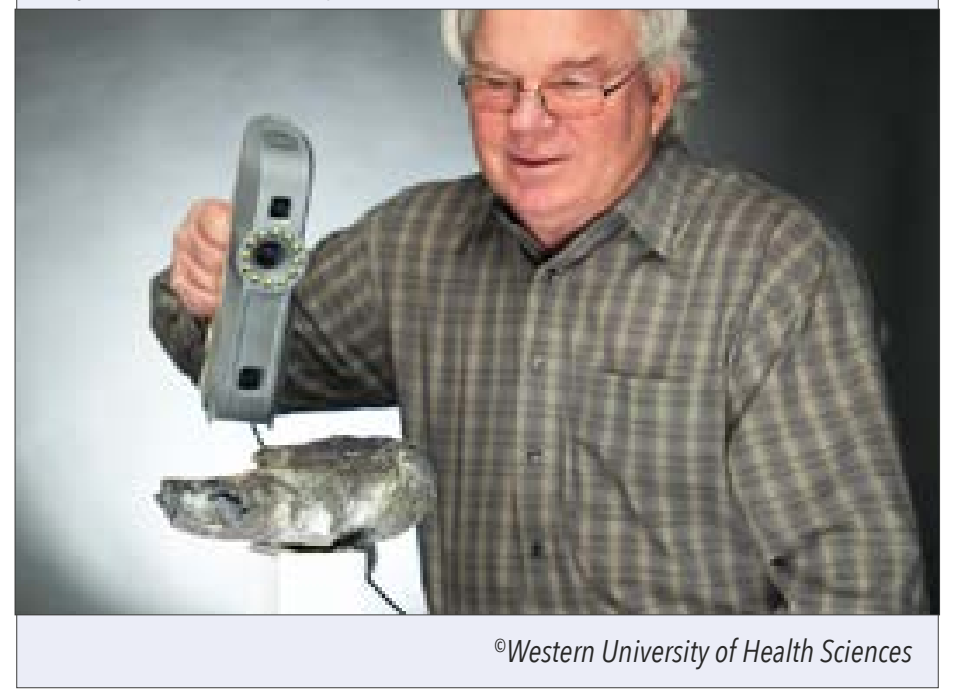

\section{METHODOLOGY}

In medical practice, 3D images can be obtained by a variety of methodologies, including 3D laser scanning, $C T$, and magnetic resonance imaging (MRI). ${ }^{3}$ In $3 \mathrm{D}$ laser scanning, the object of interest is placed on a digitizer bed. A line of laser light is projected onto the surface while two sensors record the displacement of the laser light, demonstrating the changing contour and shape of the object (Figure 1). Scanning can be performed using stationary scanners, handheld scanners, an image of which is shown below, and smartphones that have been integrated with 3D scanning technology. ${ }^{9}$

The CT scanner generates an X-ray that creates a snapshot image that is recorded by a detector on the opposing side. These cross-sectional images are taken in succession until the desired area is fully imaged, then they are combined for a $3 \mathrm{D}$ image ${ }^{3}$. In MRI, radio waves are sent by the machine with the purpose of stimulating hydrogen atoms within cells. The hydrogen atoms emit energy that is converted to numbers. The numbers are processed in a computer and an image is produced. ${ }^{3}$

3D imaging consists of 3D scanning, modeling, and printing. It is the process of producing a digital representation of an object. 3D scanning can be used to generate 3D models and $3 \mathrm{D}$ prints of an object and uses computer software to take a collection of data points from the scanned image and recreate them in a three-dimensional space. 3D printing is the process of producing a tangible object from a computer aided design model, which is often interchangeably called 
additive manufacturing as the object is formed from additive layering of material on top of material. This was traditionally done with a 3D printer, using polymer as the material, but in the recent years, different applications of 3D printing have allowed expansion to different materials, such as epoxy resins, titanium, steel, and wax. ${ }^{10}$

\section{CURRENT APPLICATIONS}

3D imaging has rapidly made its advent in multiple specialties of medical practice, including orthopedic surgery, neurovascular surgery, forensic pathology, pathology, and dentistry. It has also made an impact in medical and allied health education.

\section{Orthopedic surgery}

3D images of bones are reconstructed from a CT or $\mathrm{MRI}$, and a prototype of the bone is printed via layered manufacturing technique for surgical implantation. In addition, the prototype of the bone allows the surgeon to familiarize themselves with the procedure prior to execution. Prosthetics are also being produced with 3D imaging, modeling, and printing. With these technologies, the prosthesis can be customized to the specific needs of the patient. ${ }^{11}$ As $3 \mathrm{D}$ printers are becoming widely available, they are no longer an expensive product. Customizing prostheses for patients is more cost effective as it allows for a more detailed fit, shorter surgical duration and uneventful recovery time. $3 \mathrm{D}$ printing of the patients' bones is patient specific and enables physicians to construct prosthetics that are tailored to meet the patient's surgical needs, in terms of

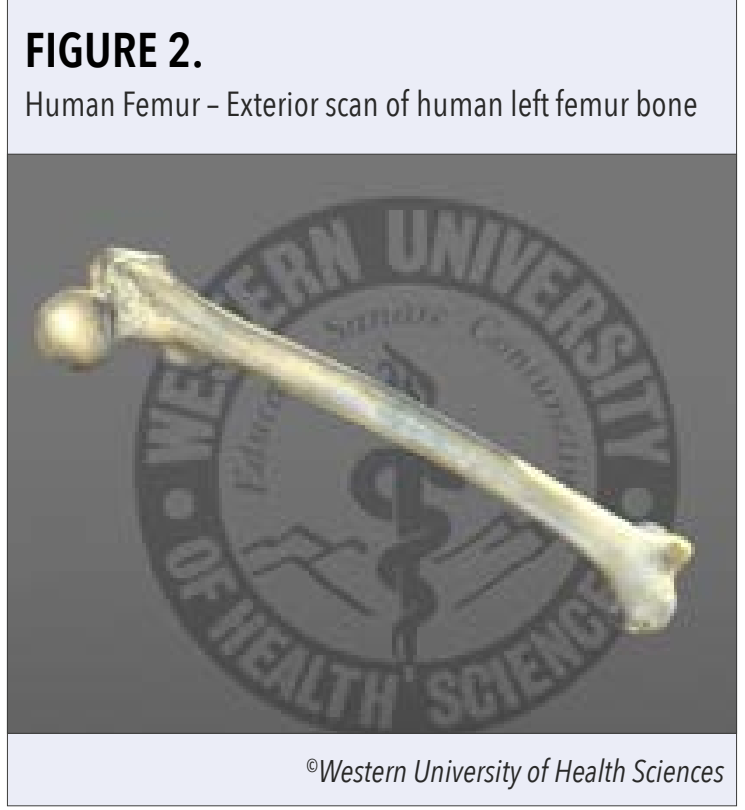

shape, size and unique anatomy (Figure 2).

\section{Neurovascular surgery}

Pre-surgical $3 \mathrm{D}$ imaging and modeling of the neurovascular abnormality can allow the surgeon to not only diagnose but also plan the surgical procedure. Sullivan et al. describes a case report in which an eight-year-old boy was treated for a fusiform aneurysm of the left internal carotid artery. Due to the complexity of the anatomy, the neurosurgeons needed a 3D model of the patient's arteries in order to develop a surgical plan. The patient's cerebral vasculature was $3 \mathrm{D}$ printed with silicone using the technology, Vascular Simulations, and the surgical plan was rehearsed through simulations. An image of the silicone 3D model is shown in Figure 3. The arrow is pointing to an aneurysm in the ICA and the arrow head is pointing to a kink in the wall of the MCA. Without 3D technology, the surgery would have taken longer, with longer period of general anesthesia, and with increased risk for error due to poor visualization of the patient's unique vascular anatomy.

\section{Forensics}

With 3D imaging, forensic investigation can map entire crime scenes and reconstruct 3D models of evidence such as bones, teeth, and fingerprints. During a crime investigation, the teeth of victims are often displaced in the midst of a disaster or accident. Digital tooth reconstruction can be used to $3 \mathrm{D}$ print a tooth with the information given by the intra-alveolar morphology of the tooth socket. ${ }^{13} \mathrm{~A}$ study by Johnson et al. has shown that there are minimal errors in the reconstruction of the 3D printed tooth. This enhances the victim identification process in forensic investigations even in the absence of teeth. ${ }^{13}$ Depicted in Figure 4 is a 
3D model of a 1st molar, tooth " 3 " according to the Dental Numbering System. Evidence of human remains, such as teeth, found at crime scenes can be reconstructed to produce models like the one shown.
Confocal scanning microscopy is a customized light microscopy which increases the depth and resolution of the tissue specimen in a three-dimensional view. ${ }^{19}$ The combinations of these two techniques provided a more sensitive visualization of the tissue specimens, such that they were able to identify microscopic metastatic lesions in the lymph nodes, previously missed by conventional light microscopy. ${ }^{20}$ Tolkach et al. explored the use of $3 \mathrm{D}$ reconstruction technology on prostate adenocarcinoma specimens and its morphology. ${ }^{16}$ Reconstruction technology uses laser scanners to algorithmically reconstruct the specimen tissue forming a three-dimensional image. ${ }^{19}$ 3D reconstruction can help with evaluating the grading score, direction of tumor growth, invasive pattern of tumor, and patient prognosis. ${ }^{16}$ 3D imaging (CBCT), the orthodontist is able to successfully treat CLP. It also allows them to see if the airway and oral structures are affected by the pathological process. ${ }^{14}$ With $\mathrm{CBCT}$, orthodontists are able to effectively plan their surgical procedures and develop bone grafts to treat CLP patients, successfully restoring normal speech and feeding functions.

\section{Pathology}

Though light microscopy is the current gold standard for tissue specimen analysis, there are many drawbacks to its use. It can be destructive to the tissue specimen, distort the tissue morphology, limit the potential to analyze the tissue's microenvironment, and can be very time consuming 16,17,18. 3D imaging technologies such as special tissue clearing techniques, laser scanners, whole slide imaging, file formats, and 3D reconstructions of tissue specimens have become powerful assets for pathologists in extending their knowledge and understanding of histopathology. ${ }^{19}$

A study from the Osaka University, Graduate School of Medicine, focused on the use of 3D confocal scanning microscopy with a tissue clearing technique called clear unobstructed brain imaging cocktail and computational analysis (CUBIC). ${ }^{20} \mathrm{CUBIC}$ tissue clearing technique is useful in enhancing the transparency of the tissue specimens.

technologies can also be used for educational purposes in pathology, enhancing the learning process for students and residents.

\section{Medical and allied health education}

The application of 3D imaging technologies has not only transformed our clinical knowledge in the healthcare field, but it has also become an important teaching aid. Medical students benefit from this technology as 3D anatomical atlases of the human body have been incorporated into the medical education, allowing students to better understand spatial anatomy. ${ }^{21}$ A randomized control study at Yuying Children's Hospital of Wenzhou Medical University, showed that 3D printed models were beneficial in helping students understand complex anatomical sites. ${ }^{22}$ It has also become a crucial training technology for surgical residents. A study using 3D imaging to stimulate a virtual reality (VR), allowed surgical residents to practice their surgical skills. The study found VR training to be helpful for surgical residents practicing procedures. ${ }^{23}$ In addition, nursing education has also implemented 3D imaging technologies as a way of improving care for their patients. Results from a study showed 3D imaging models of complex congenital heart diseases (CHD) were used to help nurses better nderstand cardiac anomalies. ${ }^{24}$ 


\section{FIGURE 5.}

Interactive 3D image of a hysterectomy specimen with a leiomyoma.

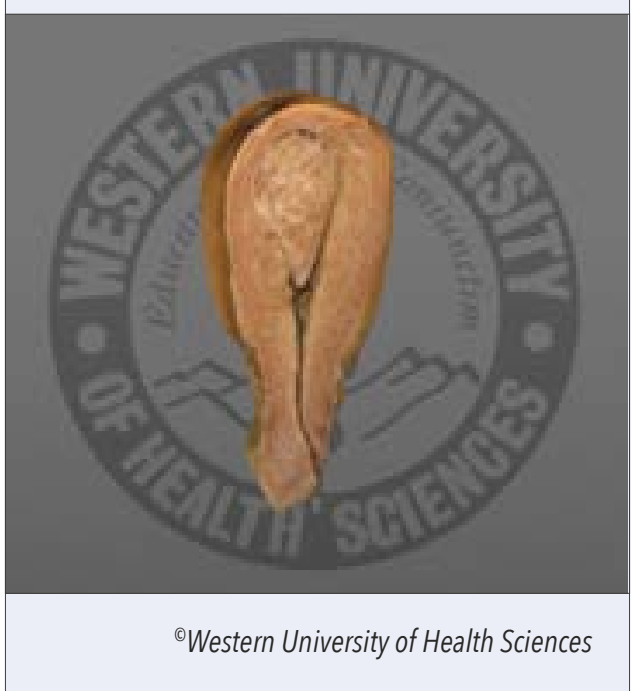

\section{FUTURE APPLICATIONS}

Given the ability to duplicate the radiologic abnormalities, $3 \mathrm{D}$ imaging has already served as a useful tool in many fields as discussed above. In the future, this technology will continue to advance radiology by increasing the diagnostic sensitivity. Given 3D technology can be used to image and print complex structural anomalies, such as valvular, and congenital disorders, it will be beneficial in depicting the patient's heart for custom implants and devices. ${ }^{25}$ The incorporation of $3 D$ imaging technologies in surgery will continue to benefit the healthcare system financially and improve surgical outcomes for patients. With the reduction in surgery time and postoperative complications, studies showed 3D imaging has helped reduce operating room cost ranging from $\$ 1488-\$ 3720$ per surgical case. ${ }^{26}$ 3D imaging technologies such as handheld intraoral scanners will allow dentists to have a quick and precise image of dental anatomy. ${ }^{27}$ The images will be used to print 3D models of orthodontic splints, night guards, and aligners, all without having to make a physical mold out alginate, a current practice seen traditionally. ${ }^{28}$

\section{CONCLUSION}

3D imaging has become a major advancement in health technologies and has shaped the health field in clinical diagnosis, treatment options, customized patient care, and health education. It is a foundation in many diagnostic procedures such as evaluating intracranial aneurysm and staging cancers. In addition, 3D imaging has made a substantial impact on treatment options for clinicians and their patients. It has helped clinicians save lives in a variety of disease entities, including valvular heart defects and congenital malformations. The technology has also benefited patients by receiving meaningful, personalized care, such as customized prostheses, thereby streamlining the surgical procedure and the postoperative progress. Furthermore, 3D imaging technologies can help decrease health care costs due to its efficient diagnostic procedures, shorter surgical duration, and effective treatment plans. In academia, 3D imaging has been used in numerous health professions to further educate medical students, nurses, and surgeons. In conclusion, 3D imaging has revolutionized medicine and its continual development will rapidly become a mainstay in diagnostics and treatments in health care.

\section{ACKNOWLEDGEMENTS}

We would like to acknowledge Gary Wisser, Educational 3D Visualization Specialist at the Center for Excellence in Teaching and Learning (CETL) for providing access to the Western University of Health Sciences collection of 3D images. Images depicted in this article can be viewed in the 3D mode at https://sketchfab.com/WesternU3D

\section{AUTHOR DISCLOSURES:}

No relevant financial affiliations or conflicts of interest.

\section{REFERENCES:}

1. Broadbent B. A new X-ray technique and its application to orthodontia. angle orthod. 1931; 45-66.

2. Kolokitha $\mathrm{O}$, and Evangelia $\mathrm{C}$. Factors influencing the accuracy of cephalometric prediction of soft tissue profile changes following orthognathic surgery. Journal of maxillofacial and oral surgery $11.12012 ; 82-90$.

3. Karatas $\mathrm{OH}$, Toy E. Three-dimensional imaging techniques: A literature review. Eur J Dent. 2014;8(1):132-140. doi:10.4103/1305-7456.126269

4. Kalender WA. X-ray computed tomography. Physics in Medicine and Biology. 2006;51(13). doi:10.1088/0031-9155/51/13/r03

5. Goldman LW. Principles of CT and CT Technology. Journal of Nuclear Medicine Technology. 2007;35)3_:115-128.doi:10.2967//jnmt.107.042978 
6. Vukicevic M, Mosadegh B, Min JK, Little SH. Cardiac 3D Printing and its Future Directions. JACC: Cardiovascular Imaging. 2017;10(2):171-184. doi:10.1016/j.jcmg.2016.12.001.

7. Lee EY, Boiselle PM, Shamberger RC. Multidetector computed tomography and 3-dimensional imaging: preoperative evaluation of thoracic vascular and tracheobronchial anomalies and abnormalities in pediatric patients. Journal of Pediatric Surgery. 2010;45(4):811-821. doi:10.1016/j.jpedsurg.2009.12.013.

8. Huang HK. PACS Research and Development A Review and Perspective. Picture Archiving and Communication Systems (PACS) in Medicine. 1991:1-7. doi:10.1007/978-3-642-76566-7_1.

9. W. J. Walecki; F. Szondy; M. M. Hilali (2008). "Fast in-line surface topography metrology enabling stress calculation for solar cell manufacturing allowing throughput in excess of 2000 wafers per hour". Meas. Sci. Technol. 19 (2): 025302.

10. Shahrubudin N, Lee TC, Ramlan R. An Overview on 3D Printing Technology: Technological, Materials, and Applications. Procedia Manufacturing. 2019; 35: 1286-1296.

11. Morongiu G, Prost R, Capone A. Use of 3D modelling and $3 \mathrm{D}$ printing for the diagnostic process, decision making and preoperative planning of periprosthetic acetabular fractures. BMJ Case Rep. 2020; 13(1).

12. Sullivan $S$, Aguilar-Salinas $P$, Santos $R$, Beier A, Hanel E. Three-dimensional printing and neuroendovascular simulation for the treatment of a pediatric intracranial aneurysm: case report. Journal of Neurosurgery: Pediatrics PED. 2018;22: 672-677.

13. Johnson A, Jani G, Pandey A, Patel N. Digital tooth reconstruction: An innovative approach in forensic odontology. J Forensic Odontostomatol. 2019; 3(37): 12-20.

14. Erten $O$, Y IImaz BN. Three-Dimensional Imaging in Orthodontics. Turk J Orthod. 2018;31(3):86-94. doi:10.5152/TurkJOrthod.2018.17041

15. Shkoukani MA, Chen M, Vong A. Cleft lip - a comprehensive review. Front Pediatr. 2013;1:53. Published 2013 Dec 27. doi:10.3389/fped.2013.00053

16. Tolkach Y, Thomann S, Kristiansen G. Three-dimensional reconstruction of prostate cancer architecture with serial immunohistochemical sections: hallmarks of tumor growth, tumour compartmentalization, and implications for grading and heterogeneity. Histopathology. 2018;72(6):1051-1059. doi:10.1111/his.13467.

17. Jansen I, Lucas M, Savci-Heijink CD, et al. Histopathology: ditch the slides, because digital and 3D are on show. World Journal of Urology. 2018;36(4):549-555. doi:10.1007/s00345-018-2202-1.
18. Baker BM, Chen CS. Deconstructing the third dimension - how 3D culture microenvironments alter cellular cues. Journal of Cell Science. 2012;125(13):3015-3024. doi:10.1242/jcs.079509.

19. Farahani N, Braun A, Jutt D, et al. Three-dimensional imaging and scanning: Current and future applications for pathology. Journal of Pathology Informatics. 2017;8(1):36. doi:10.4103/jpi.jpi_32_17.

20. Nojima S, Susaki EA, Yoshida K, et al. CUBIC pathology: three-dimensional imaging for pathological diagnosis. Scientific Reports. 2017;7(1). doi:10.1038/s41598-017-09117-0.

21. Pommert, A., Riemer, M., Schiemann, T., Tiede, U., \& Höhne, K.H. Knowledge-Based and 3D Imaging Systems in Medical Education. IFIP Congress. (1994); https://pdfs.semanticscholar. rg/6c05/9db6de55712d811da27c13a9f9382ebdc93d. pdf?_ga=2.127667533.1987329504.15803499391148848694.1580349939

22. Wu A-M, Wang K, Wang J-S, et al. The addition of $3 D$ printed models to enhance the teaching and learning of bone spatial anatomy and fractures for undergraduate students: a randomized controlled study. Annals of Translational Medicine. 2018;6(20):403-403. doi:10.21037/atm.2018.09.59.

23. Pulijala Y, Ma M, Pears M, Peebles D, Ayoub A. Effectiveness of Immersive Virtual Reality in Surgical Training-A Randomized Control Trial. Journal of Oral and Maxillofacial Surgery. 2018;76(5):1065-1072. doi:10.1016/j.joms.2017.10.002.

24. Biglino G, Capelli C, Koniordou D, et al. Use of 3D models of congenital heart disease as an education tool for cardiac nurses. Congenital Heart Disease. 2016;12(1):113-118. doi:10.1111/chd.12414.

25. Harb SC, Rodriguez LL, Vukicevic M, Kapadia SR, Little SH. Three-Dimensional Printing Applications in Percutaneous Structural Heart Interventions. Circ Cardiovasc Imaging. 2019; 12(10).

26. Ballard DH, Mills P, Duszak R, Weisman JA, Rybicki FJ, Woodard PK. Medical 3D Printing Cost-Savings in Orthopedic and Maxillofacial Surgery: Cost Analysis of Operating Room Time Saved with 3D Printed Anatomic Models and Surgical Guides. Academic Radiology. 2019. doi:10.1016/j.acra.2019.08.011.

27. Winkler J, Gkantidis N. Trueness and precision of intraoral scanners in the maxillary dental arch: an in vivo analysis. Sci Rep. 2020; 10(1).

28. Taneva E, Kusnoto B, Evans C. 3D scanning, imaging, and printing in orthodontics. Issues in contemporary orthodontics. 2014. DOI: 10.5772/60010. 